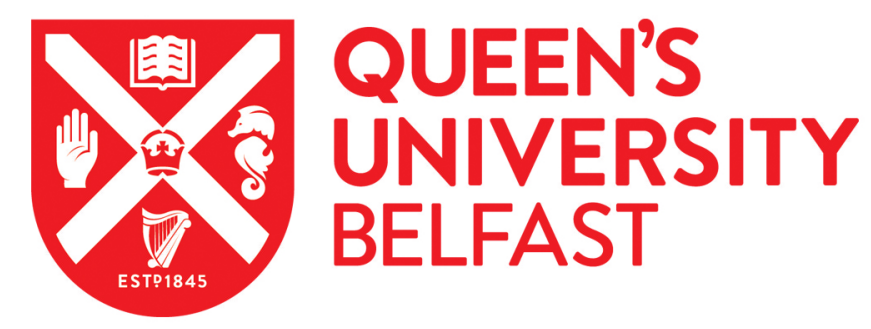

\title{
Conventional in vivo irradiation procedures are insufficient to accurately determine tumor responses to non-uniform radiation fields
}

\author{
Butterworth, K. T., Redmond, K. M., McMahon, S. J., Cole, A. J., Jain, S., McCarthy, H. O., O'Sullivan, J. M., \\ Hounsell, A. R., \& Prise, K. M. (2015). Conventional in vivo irradiation procedures are insufficient to accurately \\ determine tumor responses to non-uniform radiation fields. International journal of radiation biology, 91(3), 257- \\ 261. https://doi.org/10.3109/09553002.2014.980468
}

Published in:

International journal of radiation biology

Document Version:

Peer reviewed version

Queen's University Belfast - Research Portal:

Link to publication record in Queen's University Belfast Research Portal

Publisher rights

(C) 2014 Informa UK, Ltd.

This is an Accepted Manuscript of an article published by Taylor \& Francis in International Journal of Radiation Biology on 20 Nov 2014 , available online: http://www.tandfonline.com/10.3109/09553002.2014.980468.

\section{General rights}

Copyright for the publications made accessible via the Queen's University Belfast Research Portal is retained by the author(s) and / or other copyright owners and it is a condition of accessing these publications that users recognise and abide by the legal requirements associated with these rights.

Take down policy

The Research Portal is Queen's institutional repository that provides access to Queen's research output. Every effort has been made to ensure that content in the Research Portal does not infringe any person's rights, or applicable UK laws. If you discover content in the Research Portal that you believe breaches copyright or violates any law, please contact openaccess@qub.ac.uk. 
Conventional in vivo irradiation procedures are insufficient to accurately determine tumor responses to non-uniform radiation fields

\section{Running title: In vivo responses to non-uniform radiation fields}

K. T. Butterworth ${ }^{\mathrm{a}}$, K. M. Redmond ${ }^{\mathrm{a}}$, S.J. McMahon ${ }^{\mathrm{a}}$, A. J. Cole ${ }^{\mathrm{b}}$, H. O. McCarthyc, J. M. O’Sullivan ${ }^{\mathrm{b}}$, A. R. Hounsell ${ }^{\mathrm{d}}$, K. M. Prise ${ }^{\mathrm{a}}$.

a Centre for Cancer Research and Cell Biology, Queen’s University Belfast, Belfast, Northern Ireland, UK; ${ }^{\mathrm{b}}$ Clinical Oncology, Northern Ireland Cancer Centre, Belfast Health and Social Care Trust, Belfast, Northern Ireland, UK; c School of Pharmacy, Queen’s University Belfast, Belfast, Northern Ireland, UK; 'd Radiotherapy Physics, Northern Ireland Cancer Centre, Belfast Health and Social Care Trust, Northern Ireland, UK.

\section{Corresponding Author}

Dr Karl Butterworth

Centre for Cancer Research and Cell Biology, Queen’s University Belfast, 97 Lisburn Road, Belfast BT9 7BL, Northern Ireland, United Kingdom.

Tel: $+44(0) 2890972945$

E-mail: kbutterworth@qub.ac.uk

Keywords; bystander, bioluminescent imaging, non-uniform, in vivo, tumour burden 


\section{ABSTRACT}

Purpose: To determine differences in overall tumor responses measured by volumetric assessment and bioluminescence imaging (BLI) following exposure to uniform and nonuniform radiation fields in an ectopic prostate tumour model.

Materials and Methods: Bioluminescent human prostate tumor xenografts were established by subcutaneous implantation into male mice. Tumors were irradiated with uniform or non-uniform field configurations using conventional in vivo irradiation procedures performed using a $225 \mathrm{kVp}$ generator with custom lead shielding. Tumor responses were measured using Vernier calipers and by BLI using an in vivo imaging system. Survival was defined as the time to quadroupling of pre-treatment tumor volume. Results: The correlation between BLI and tumor volume measurements was found to be different for un-irradiated $(R=0.61)$, uniformly irradiated $(R=0.34)$ and partially irradiated $(R=0.30)$ tumors. Uniformly irradiated tumors resulted in an average tumour growth delay of 60 days with median survival of 75 days, compared to partially irradiated tumors which showed an average growth delay of 24 days and median survival of 38 days.

\section{Conclusions:}

Correlation between BLI and tumor volume measurements is lower for partially irradiated tumors than those exposed to uniform dose distributions. The response of partially irradiated tumors suggests non-uniformity in response beyond physical dose distribution within the target volume. Dosimetric uncertainty associated with conventional in vivo irradiation procedures prohibits their ability to accurately determine tumor response to non-uniform radiation fields and stresses the need for image guided small animal radiation research platforms. 


\section{INTRODUCTION}

Small animal models are a fundamental tool for robust preclinical radiobiological investigations. Technological advances in the clinic, such as intensity modulated and image guided radiotherapy, have significantly outpaced those for the irradiation of laboratory animals under experimental conditions which has commonly been performed using fixed kilovoltage sources with custom lead shielding for beam targeting and sparing of normal tissues (Hillman et al, 2001; Know et al, 1992).

This translational gap between experimental radiobiology and radiation oncology has been narrowed with the implementation of small animal image guided radiation research platforms (Verhaegen et al, 2011; Zhou et al, 2010; Wong et al, 2008) which combine high resolution cone beam computed tomography (CBCT) with orthovoltage radiation sources for accurate beam targeting allowing treatment of a defined target through a process analogous to that of contemporary clinical practice.

The implementation of these platforms in the laboratory represents a significant advancement for in vivo radiobiological investigations enabling preclinical evaluation of novel regimes including hypofractionation, dose painting and drug radiation combinations. Furthermore, the development of small animal radiation research platforms with on board optical imaging will offer significant potential in radiation biology.

A range of optical imaging and spectroscopy methods are widely used for radiobiological studies to determine cellular responses at the molecular level (Palmer et al, 2012). Bioluminescence imaging (BLI) is a highly sensitive, non-invasive in vivo imaging modality which measures photons generated by a luciferase reporter gene in the presence of the D-luciferin substrate injected prior to imaging (Inouye et al, 2010). BLI has been used to assess tumour burden in a range of preclinical models for localised and metastatic 
disease (O’Neill et al, 2010; Tuli et al, 2012; Al Nakouzi et al, 2012), however, its application in monitoring the efficacy of radiation exposure responses has been limited (Lee et al, 2010).

In the present study, we aimed to compare tumour volume and BLI measurements to determine differences in overall tumor response following exposure to uniform and nonuniform radiation fields in an ectopic prostate tumor model. This study also sought to provide in vivo support for the presence of out-of-field effects under non-uniform dose distributions.

\section{MATERIALS AND METHODS}

Cell culture

PC-3-luc2 Bioware Ultra (Caliper Life Sciences, Runcorn, United Kingdom) is a stably transfected luciferase expressing variant of the human prostate cancer cell line PC-3 (Kaighn et al, 1979). Cells were grown in RPMI (Roswell Park Memorial Institute)-1640 medium with 10\% fetal bovine serum, 1\% penicillin / streptomycin (Gibco, Paisley, Scotland, UK) and maintained at $37^{\circ} \mathrm{C}$ in a humidified atmosphere of $95 \%$ air $/ 5 \% \mathrm{CO}_{2}$.

\section{Animals, tumor implantation and maintenance}

6-8 week old male Fox Chase SCID (Severe combined immunodeficient) mice (Charles River Laboratories, Oxford, United Kingdom) were used as a xenograft model for PC-3luc2 cells. $1.5 \times 10^{6}$ cells in $100 \mu$ of PBS (phosphate buffered saline) were implanted intra-dermally on to the flank of animals under inhalant anaesthesia. 5 animal were assigned to each experimental group (control, uniform irradiated, partially irradiated). Animals received food and water ad libitum. All experimental procedures were carried 
out in accordance with United Kingdom Home office approved protocols for in vivo experimentation.

\section{Irradiation procedure}

Tumors were grown for 4-8 weeks until reaching a pre-treatment volume of around 100 $\mathrm{mm}^{3}$. Animals were assigned to three treatment groups, un-irradiated controls, $8 \mathrm{~Gy}$ irradiated animals delivered as a uniform field and 8 Gy irradiated animals delivered as non-uniform field in which $40-60 \%$ of the tumor volume was irradiated. Animals were restrained and custom lead shielding used to expose the complete or partial tumour volume to $225 \mathrm{kVp}$ X-rays using a X-Rad 225 generator (Precision X-ray Inc, North Branford, Connecticut, USA). A dose profile was generated using Gafchromic RTQA2 film (Ashland, Covington, Kentucky, USA) placed under the lead shielding which show scattered dose fell to less than $10 \%$ of the target dose within $1 \mathrm{~cm}$ off axis as shown in figure 1 . Animals were irradiated at a dose rate of $0.52 \mathrm{~Gy} \mathrm{~min}^{-1}$.

\section{Tumour volume measurements and BLI}

Tumor volume was determined three times a week from Vernier caliper measurements in three orthogonal dimensions. Tumour bearing mice were imaged weekly using an IVIS 100 (Caliper Life Sciences). Animals were intraperitoneally injected with Dluciferin potassium salt (Caliper Life Sciences) at a concentration of $150 \mathrm{mg} / \mathrm{kg}$ and imaged 15 minutes after injection as determined from BLI kinetic studies in the same tumor model. The distribution of detected photons was overlaid onto a grayscale photographic image and a region of interest (ROI) manually selected. Signal intensity was quantified in photons/second/squared centimetre/steradian $\left(\mathrm{p} / \mathrm{s} / \mathrm{cm}^{2} / \mathrm{Sr}\right)$ for a ROI 
selected manually by applying the same threshold to each of the images using Living Image Software (Caliper Life Sciences).

\section{Statistical Analysis}

The correlation between tumour volume and BLI measurements was assessed using a linear regression of the form $y=m x+c$, where $x$ is the measured volume, $y$ the BLI value, $m$ the slope and $c$ the $y$-intercept of the linear fit of the data. Significant correlation was evaluated using a two-tailed t test on $m$, with a significance threshold of 0.05. All calculations were carried out using (GraphPad Prism, Version 5.01).

\section{RESULTS}

\section{Correlation of tumor volume and BLI measurements}

Correlation of tumor volume and BLI measurements was determined for control, uniformly irradiated and partially irradiated tumours in a heterotopic prostate xenograft model. Photon emission from a manually selected region of interest showed maximum flux at 15 - 20 minutes after intraperitoneal injection of D-luciferin in animals pretreatment. Control and irradiated animals showed no significant difference in the kinetics of bioluminescence signal at the beginning of the experiment or when maximum tolerated tumour volume was reached at the end of the experiment (data not shown).

Comparison of BLI and tumour volume for control, uniformly irradiated and partially irradiated tumours is shown in figure 2. Linear regression analysis showed significant correlation between tumor volume and BLI for control tumors $(R=0.6123)$. A less strong correlation between tumor volume and BLI was observed for uniformly irradiated tumours $(R=0.3417)$ and the least significant correlation shown for partially irradiated tumors (40 $-60 \%$ tumour volume; $R=0.3063$ ). Significance testing of these correlation 
coefficients using a t-test indicated statistically significant correlation for control $(\mathrm{p}=$ $0.01)$ and uniformly irradiated animals $(p=0.02)$ but no significant relationship for partially exposed tumors $(\mathrm{p}=0.08)$.

Tumor growth delay and overall survival

Tumor volume measurements are shown for control, uniformly irradiated and partially irradiated animals in figure 3a. Comparing the data for control with uniformly irradiated tumours showed a significant growth delay of around 60 days which is reflected in the

median survival of 14 and 75 days respectively as shown in figure 3b. Comparison of control with partially uniformly irradiated tumours showed a significant growth delay of around 24 days which is reflected in the median survival of 14 and 38 days respectively as shown in figure 3b. The mean fractional uncertainty in tumor volume measurements was found to be 0.12 for control animals compared to 0.26 for partially irradiated and 0.24 for uniformly irradiated animals.

\section{DISCUSSION}

This study aimed to determine differences in the overall tumor response measured by volumetric assessment and BLI in un-irradiated and irradiated tumors exposed to uniform or non-uniform radiation fields. These experiments were conducted in the context of assessing BLI as a means to determine the effective spatial dependency of radiobiological effects occurring outside of the primary treatment field and to assess if this could be achieved using convention in vivo irradiation configurations.

Tumors were implanted intra-dermally to minimise physical limitations of tissue depth, photon signal impedance and to improve tumour target definition. As the kinetics of luciferase is an important consideration in BLI studies, this was determined in animals pre- and post-irradiation. In agreement with previous reports (Kemper et al 2006; 
Burgos et al, 2003), maximum photon flux was observed 15 minutes post injection for both the control and irradiated animals. Time taken for acquisition of BLI was comparable to that of caliper measurements with both taking less than 1 minute.

BLI is a powerful non-invasive tool for imaging of tumor burden in small animals (Klerk et al, 2007). Consistent with other reports (Klerk et al, 2007; Paroo et al, 2004), significant correlation between bioluminescence and tumour volume was observed for control tumours $(R=0.6123, \mathrm{p}=0.01)$ indicating utility of BLI for quantitative assessment of tumour burden. Correlation between these measurements decreased for uniformly irradiated tumours $(R=0.3417, \mathrm{p}=0.02)$ suggesting BLI is less accurate in predicting volume for irradiated ectopic tumours. We postulate that the observed differences in predictive power between control and irradiated tumours is a consequence of inaccuracies associated with caliper measurements as areas of necrosis and oedema may be measured in addition to viable tumour cells. In addition, similar discrepancy between BLI signal and tumor volume measured by magnetic resonance (MR) was reported by Jost et al, (2009) in an orthotopic brain tumor model showing poor correlation with very low BLI signal for very large tumor volumes.

There may be significant biological heterogeneity in factors impacting on radiation response such as radiation induced cell death, damage to the tumour vascular damage and surrounding normal tissue and regions of hypoxia. These factors may also in part explain the poor correlation observed in partially irradiated tumours $(R=0.3063, \mathrm{p}=$ 0.08). Given the assumption of symmetrical tumors when using orthogonal measurements to calculate tumour volume, the relationship may be unsurprising. BLI may be a more accurate means of determining response to partial radiation fields where asymmetrical tumor volumes may result. 
Considering the differences in dose distributions between partially and uniformly irradiated tumors, this may also suggest a significant underlying biological component in response similar to the out-of-field effects observed in vitro in several studies from our laboratory (Trainor et al, 2012; Butterworth et al, 2011). Notably, the growth delay seen in partially-irradiated tumours (roughly 3 doubling times) is greater than would be expected even for complete killing of the irradiated portion of the tumour, this indicates a significant reduction in viability for out-of-field cells also. Further interpretation of the data from this study in the context of out-of-field effects is limited as the low spatial resolution of both the irradiation technique and BLI may be insufficient to detect significant variation in response occurring out-of-field.

An improved approach may be the combination of BLI with accurate dose distributions offered through small animal image guided radiotherapy platforms. Furthermore, the development of tomographic optical imaging systems allowing the acquisition of BLI from multiple angles with reconstruction algorithms applying models of photon transport is likely to offer improved resolution in three dimensions. Although ectopic tumor models remain important experimental tools in biological sciences they fail to accurately recapitulate the tumour microenvironment in situ. To investigate tumour development and response to complex radiation fields delivered typically during advanced radiotherapy, the application of orthotopic tumour models may be more biologically relevant in recapitulating the tumor microenvironment.

In summary, the data in this study show a clear correlation between tumor volume and BLI intensity. However, there may be significant inter-tumoral and inter-animal variations which may impact on absolute signal intensity particularly following irradiation. This study shows conventional in vivo radiation procedures using lead shielding are insufficient in accurately determining tumor responses to non-uniform 
radiation fields, stressing the need for the application of more accurate image guided small animal irradiation platforms in such investigations.

\section{ACKNOWLEDGEMENTS}

We would like to acknowledge Dr Conor McGarry at the Northern Ireland Cancer Centre for his useful insight concerning this project. This work is supported by Cancer Research UK (C1513/A7047 to KMP and C212/A11342 for ARH) and Friends of The Cancer Centre (Registered Charity NI00080).

\section{DECLARATION OF INTERESTS}

The authors report no declarations of interest.

\section{REFERENCES}

Al Nakouzi N, Bawa O, Le Pape A, Lerondel S, Gaudin C, Opolon P, Gonin P, Fizazi K, Chauchereau A. 2012. The IGR-CaP1 xenograft model recapitulates mixed osteolytic/blastic bone lesions observed in metastatic prostate cancer. Neoplasia 14:376387.

Burgos JS, Rosol M, Moats RA, Khankaldyyan V, Kohn DB, Nelson MD Jr, Laug WE. 2003. Time Course of Bioluminescent Signal in Orthotopic and Heterotopic Brain Tumors in Nude Mice. Biotechniques 34:1184-1188.

Butterworth KT, McGarry CK, Trainor C, O'Sullivan JM, Hounsell AR, Prise KM. 2011. Out-of-field cell survival following exposure to intensity-modulated radiation fields. Int J Radiat Oncol Biol Phys. 79:1516-1522. 
Hillman GG, Maughan RL, Grignon DJ, Yudelev M, Rubio J, Tekyi-Mensah S, Layer A, Che M, Forman JD. 2001. Neutron or photon irradiation for prostate tumors: enhancement of cytokine therapy in a metastatic tumor model. Clin Cancer Res 7:136144.

Inouye S. 2010. Firefly luciferase: an adenylate-forming enzyme for multicatalytic functions. Cell Mol Life Sci 67:387-404.

Kaighn ME, Narayan KS, Ohnuki Y, Lechner JF, Jones LW. 1979. Establishment and characterization of a human prostatic carcinoma cell line (PC-3). Invest Urol 17:16-23.

Kemper EM, Leenders W, Küsters B, Lyons S, Buckle T, Heerschap A, Boogerd W, Beijnen JH, van Tellingen O. 2006. Development of luciferase tagged brain tumour models in mice for chemotherapy intervention studies. Eur J Cancer 42:3294-3303.

Klerk CP, Overmeer RM, Niers TM, Versteeg HH, Richel DJ, Buckle T, Van Noorden CJ, van Tellingen O. 2007. Validity of bioluminescence measurements for noninvasive in vivo imaging of tumor load in small animals. BioTechniques 43:S7-S13.

Jost SC, Collins L, Travers S, Piwnica-Worms D, Garbow JR. 2009. Measuring brain tumor growth: combined bioluminescence imaging-magnetic resonance imaging strategy. Mol Imaging 8(5):245-253.

Knox SJ, Goris ML, Wessels BW. 1992. Overview of animal studies comparing radioimmunotherapy with dose equivalent external beam irradiation. Radiother Oncol 23:111-117.

Lee CJ, Spalding AC, Ben-Josef E, Wang L, Simeone DM. 2010. In vivo bioluminescent imaging of irradiated orthotopic pancreatic cancer xenografts in nonobese diabetic-severe combined immunodeficient mice: a novel method for targeting and assaying efficacy of ionizing radiation. Transl Oncol 3:153-159. 
O'Neill K, Lyons SK, Gallagher WM, Curran KM, Byrne AT. 2010. Bioluminescent imaging: a critical tool in pre-clinical oncology research. J Pathol 220:317-327.

Palmer GM, Vishwanath K, Dewhirst MW. 2012. Application of optical imaging and spectroscopy to radiation biology. Radiat Res 177:365-375.

Paroo Z, Bollinger RA, Braasch DA, Richer E, Corey DR, Antich PP, Mason RP. 2004. Validating bioluminescence imaging as a high-throughput, quantitative modality for assessing tumor burden. Mol Imaging 3:117-124.

Stojadinovic S, Low DA, Hope AJ, Vicic M, Deasy JO, Cui J, Khullar D, Parikh PJ, Malinowski KT, Izaguirre EW, Mutic S, Grigsby PW. 2007. MicroRT-small animal conformal irradiator. Med Phys. 34:4706-4716.

Trainor C, Butterworth KT, McGarry CK, McMahon SJ, O'Sullivan JM, Hounsell AR, Prise KM. 2012. Cell survival responses after exposure to modulated radiation fields. Radiat Res. 177:44-51.

Verhaegen F, Granton P, Tryggestad E. 2011. Small animal radiotherapy research platforms. Phys Med Biol 56:R55-83.

Tuli R, Surmak A, Reyes J, Hacker-Prietz A, Armour M, Leubner A, Blackford A, Tryggestad E, Jaffee EM, Wong J, Deweese TL, Herman JM. 2012. Development of a novel preclinical pancreatic cancer research model: bioluminescence image-guided focal irradiation and tumor monitoring of orthotopic xenografts. Transl Oncol 5:77-84.

Wong J, Armour E, Kazanzides P, Iordachita I, Tryggestad E, Deng H, Matinfar M, Kennedy C, Liu Z, Chan T, Gray O, Verhaegen F, McNutt T, Ford E, DeWeese TL. 2008. High-resolution, small animal radiation research platform with x-ray tomographic guidance capabilities. Int J Radiat Oncol Biol Phys. 71:1591-1599.

Zhou H, Rodriguez M, van den Haak F, Nelson G, Jogani R, Xu J, Zhu X, Xian Y, Tran PT, Felsher DW, Keall PJ, Graves EE. 2010. Development of a micro-computed 
tomography-based image-guided conformal radiotherapy system for small animals. Int $\mathbf{J}$ Radiat Oncol Biol Phys 78:297-305. 